\title{
La construcción de la identidad y los procesos de patrimonialización, un nuevo modelo educativo desde el enfoque de la educación patrimonial
}

Sofia Marin smarincepeda@hotmail.com Universidad de Valladolid, España

Reference

Marin, Sofia; (2012) "La construcción de la identidad y los procesos de patrimonialización, un nuevo modelo educativo desde el enfoque de la educación patrimonial", p. 88-92 . In: Barbosa, Helena; Quental, Joana [Eds]. Proceedings of the 2nd International Conference of Art, Illustration and Visual Culture in Infant and Primary Education. São Paulo: Blucher, 2015. ISSN 2318-695X, ISBN: 978-989-98185-0-7 DOI 10.5151/edupro-aivcipe-18

En la presente comunicación abordamos los resultados derivados de nuestra investigación dirigida por la Dra. Fontal Merillas desde la Universidad de Valladolid, en el marco del Proyecto de I+D+i Nacional OEPE1': Observatorio de Educación Patrimonial en España. Se trata de un estudio en profundidad del estado de la Educación Patrimonial y sus prácticas que constituye el punto de partida de nuestra investigación actual (Tesis Doctoral), donde la educación patrimonial se perfila como el enfoque más apropiado para trabajar en museos de arte contemporáneo. Para ello definimos un nuevo enfoque educativo propio, apoyándonos en los procesos de patrimonialización (Fontal, 2003) singulares y plurales, así como las formas de aprehender el arte contemporáneo como potencial patrimonio, en el complejo proceso de definición de identidades desde la infancia y ampliando su aplicación a todo tipo de destinatarios.

In this communication we present the results of our research, led by Dra. Fontal Merillas from the University of Valladolid, in the framework of the national research project OEPE: Observatory of Heritage education in Spain. It's an in-depth study of the state about practices in heritage education, configured as the starting point of our current research (Doctoral Thesis), where heritage education is emerging as the most proper focus for work in museums of contemporary art with all kinds of groups, through a new education model that relies on singular and social processes of "patrimonialización" (Fontal, 2003) as well as the ways of learning contemporary art as potential heritage, in the complex process of definition of identities with all kinds of collectives.

Artistic and heritage education, identity, museum, diversity

\section{Introducción}

El ser humano hace cultura, humaniza la realidad y la sociedad de la cual todos formamos parte. Todos somos personas con diversidad de capacidades. Esta meta-reflexión acerca de nuestra propia diversidad es una constante en la historia del ser humano y, en consecuencia, en los sistemas educativos, defendiendo que el ejercicio de la educación debe capacitar al individuo para el pleno uso de su libertad y autonomía.

Diversidad, patrimonio cultural, educación y accesibilidad son los cuatro ejes que caracterizan nuestro punto de partida, y a partir de los cuales nos formulamos una serie de preguntas que invitan a la reflexión; ¿Se está cumpliendo la igualdad en el acceso a nuestro patrimonio?, 
$2^{\text {nd }}$ International Conference Art, Illustration and Visual Culture in Infant and Primary Education

\section{Identidad-es. Posicionamiento conceptual y detección de la realidad para el retorno a \\ la normalidad.}

Figura 1. Esquema general de la investigación.
¿Se llevan a la práctica los planteamientos educativos que se basan en la normalización, la no segregación, la igualdad de oportunidades y la inclusión educativa?, ¿Se están cumpliendo los criterios de accesibilidad universal y diseño para todos en el ámbito educativo referido al patrimonio?

No se trata tanto de dar respuesta a estas preguntas, sino de utilizarlas como guía para orientar nuestra filosofía patrimonial y nuestro trabajo de investigación.

La diversidad y la accesibilidad son un aspecto transversal en nuestro pensamiento, reflejado en nuestra investigación. En la sociedad actual existe un planteamiento de normalización y respeto a los derechos humanos que va más allá del ámbito educativo, a través de leyes, planes, decretos y normas, en orden a conseguir la igualdad de oportunidades para todos.

Tomando en consideración todo esto, y desde la perspectiva del paradigma humanísticointerpretativo, centramos nuestro interés en el estudio de los significados de las acciones humanas. La accesibilidad universal a la educación, en el caso que nos ocupa, patrimonial, hoy en día, no es un hecho conseguido; Por ello buscamos, inventariamos y analizamos programas educativos significativos de educación artística y patrimonial que tengan en cuenta criterios de diseño accesible para todos, para poder así definir modos de hacer, modelos educativos y estándares de calidad en este campo. Desde el Observatorio recogemos más de mil programas y propuestas de educación patrimonial en España, estudio que nos permite conocer las acciones que se están poniendo en marcha en los últimos diez años, analizando las pautas metodológicas que siguen para detectar grietas, brechas, y puntos de unión que sirven de camino para crear lazos, a través de la definición de nuestro propio modelo educativo. Para ello hemos seguido una metodología de investigación basada en la evaluación de programas, a través de dos métodos: un exhaustivo análisis estadístico descriptivo de más de doscientos programas de educación desarrollados a nivel nacional, y una evaluación de programas en profundidad a través de una ficha de análisis inspirada en los postulados de grandes referente en el estudio de casos.

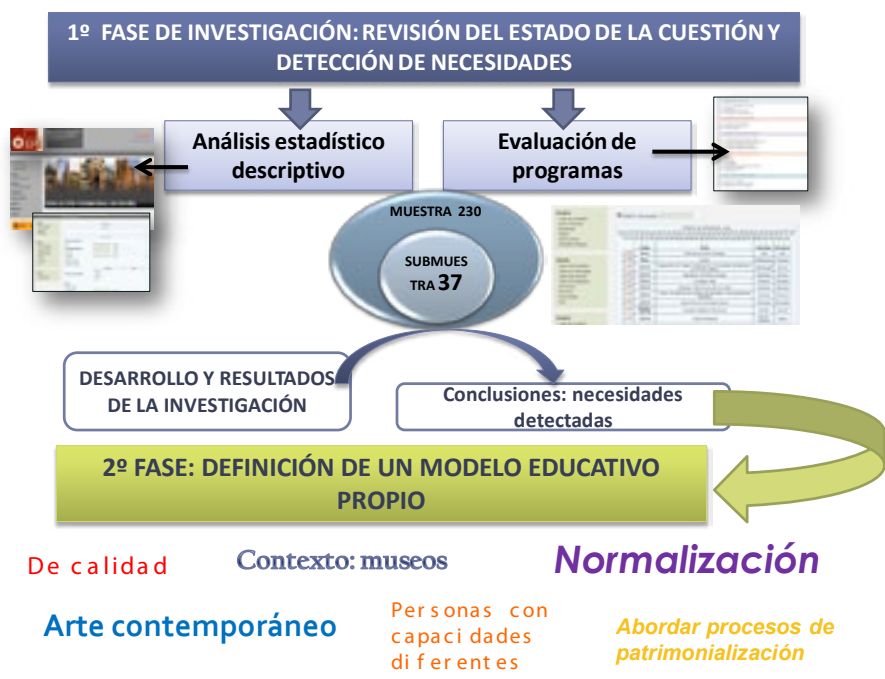

\section{Rupturas realidad-} práctica
Desde el Observatorio y a través del uso de esta potente herramienta, derivado de la primera fase de investigación descrita, constatamos que existe un contraste significativo entre las 
$2^{\text {nd }}$ International Conference Art, Illustration and Visual Culture in Infant and Primary Education $2^{\circ}$ Congreso Internacional

Arte, Ilustración y Cultura Visual en Educación Infantil y Primaria
Congresso Internacional

de Arte, Ilustração e Cultura Visual

na Educação Infantil e Primária
Figura 2. Muestra gráfica de algunos de los resultados más relevantes del estudio. demandas sociales actuales, reflejadas en las leyes y reclamadas por expertos, y la realidad de la práctica en torno a la educación patrimonial y la accesibilidad-normalización.

Localizamos 12 modelos diferentes de metodologías, que se diferencian por su diseño educativo, su estructura y sus objetivos. Los programas educativos destacan por presentar un mayor porcentaje de incidencia (21,5\%). En un segundo nivel encontramos las herramientas educativas y proyectos de mejora (13,5\% en cada caso), de lo que se puede deducir que es más frecuente encontrar proyectos que buscan mejorar o adaptar otros ya existentes, que proyectos o programas de nueva creación y con intención de generar prácticas normalizadas y estables. Sólo un 5,4\% son proyectos de investigación, un porcentaje bajo en relación con la necesidad de innovación en este campo.



De los programas que abordan la accesibilidad, un 35\% se centra en el patrimonio cultural, seguido de "Lugares creados por el hombre y la naturaleza" (18,9\%). Esto significa que sí existe la conciencia de adaptar y hacer accesible el patrimonio cultural para atender a la diversidad, en cambio, no encontramos programas que aborden otros tipos de patrimonio, como el digital, o los lugares arqueológicos.

Las capacidades diferentes para las que hemos encontrado mayor número de programas es la sensorial, visual y auditiva (29,7\%). En la misma proporción localizamos programas para discapacidad física. Es importante destacar que el mayor porcentaje localizado de programas que abordan las capacidades diferentes no especifican qué tipo de diversidad funcional abordan (35,1\%). Además un 13,5\% se refiere a "todas las discapacidades", sin especificar la tipología específica a la que se refiere, ni qué clasificación orienta esta afirmación. No todos los programas plantean objetivos didácticos. En 64,86\% de los casos sí persiguen objetivos claramente didácticos, casos en los que concluimos que se trata de objetivos de accesibilidad, difusión, mejora y análisis, de lo que se deduce un interés relacionado con la mejora y el acceso a lo educativo, vinculado indirectamente a ese carácter didáctico que sí encontramos en el resto de programas.

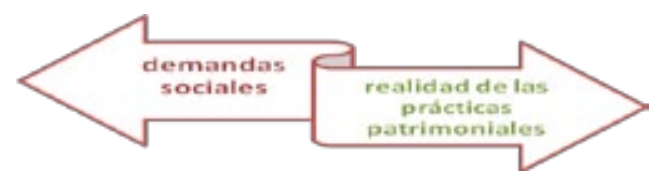

Por ello, estimamos necesario plantear y buscar soluciones al problema de investigación hallado: el conocimiento y la mejora de las prácticas en este campo a través de un nuevo y único modelo educativo de calidad que nos permita trabajar los procesos de patrimonialización en la igualdad de la diferencia.

Retomando las hipótesis planteadas en nuestra investigación verificamos que, efectivamente, existe una gran dispersión de programas de educación patrimonial y accesibilidad, para los 
$2^{\text {nd }}$ International Conference Art, Illustration and Visual Culture in Infant and Primary Education $\circ$ Congresso Internacional

de Arte, Ilustração e Cultura Visual

na Educação Infantil e Primária

\section{Mío, tuyo, suyo, nuestro. Un modelo de patrimonialización para todos.}

Figura 4. Esquema evolutivo de la investigación. cuales no se ha definido un marco teórico uniforme ni consensuado para el trabajo desde criterios de accesibilidad. No obstante, encontramos patrones, líneas y criterios en estos programas educativos que nos permiten definir modelos y tipologías. De esta manera concluimos que no existe un modelo educativo definido en materia de patrimonio, educación y accesibilidad universal. Por último, verificamos la hipótesis que sostiene que no se están cumpliendo los criterios de accesibilidad que sí aparecen recogidos en los marcos legislativos, tanto a nivel autonómico como a nivel nacional.

Observamos que, cuando se habla de accesibilidad, la mayoría de las veces se hace en referencia al acceso físico a un lugar. Pero en la educación, el estar en un lugar no implica necesariamente procesos de enseñanza-aprendizaje. Cuando hablamos de accesibilidad nos referimos a todas sus esferas, la intelectual, la sensorial, entre muchas otras. No obstante, al margen de los intereses subyacentes, todo intento, creemos que es un pequeño avance hacia esa meta común de acceso universal. Por eso consideramos de gran importancia que estas prácticas se apoyen en criterios de calidad, sin correr el peligro de subrayar de nuevo la diferencia a través de prácticas aisladas y segregadas, para lograr una normalización real y óptima en la educación patrimonial.

A raíz de este profundo análisis y del conocimiento de la situación de partida, extraemos las conclusiones e ideas que orientan la investigación actualmente hacia nuevos horizontes, y que presentamos en a continuación.

El papel de la educación, en relación con el la expresión artística y el patrimonio, es hacer efectivo todo su potencial identitario, conocerlo, comprenderlo y valorarlo. En ese camino de apertura social del patrimonio hay que tener en cuenta un diseño para todos. Esto no es un adaptar para las discapacidades, sino lograr un diseño único, válido para todos, del que todos nos beneficiemos. Se trata de proporcionar un uso sencillo y equitativo para todos. Por esto, para su cumplimiento, definimos un nuevo modelo educativo que se basa en el respeto por la diferencia y su aplicabilidad con todo tipo de colectivos.

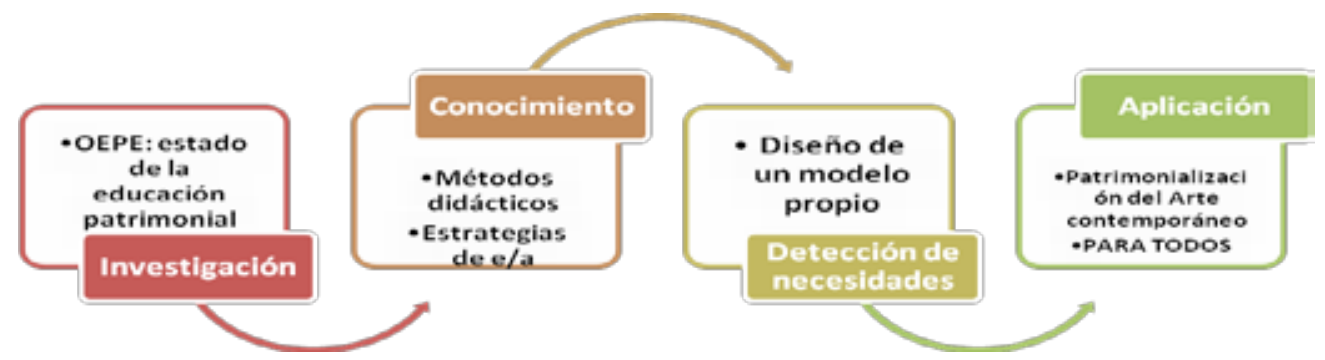

En esencia, el enfoque y modelo educativo que definimos se caracteriza por la comprensión del arte contemporáneo como potencial patrimonio cultural de la sociedad en el momento presente, y como la herramienta con la cual, a través de la educación patrimonial y la patrimonialización, trabajar aspectos clave como son identidad, propiedad y pertenencia, con todo tipo de colectivos.

Una época sólo puede ser reproducida por sus artistas, quiero decir por los artistas que viven en ese período. Creo que los artistas de una época son incapaces de reproducir el carácter de una época pasada o futura, es decir, de pintar el pasado o el futuro.²

La patrimonialización, la propiedad y los vínculos afectivo/emocionales con determinados bienes y objetos son universales en el ser humano, independientemente de la raza, cultura, origen, cualidades, edades y características del sujeto. Patrimonializar significa, según nuestro enfoque, establecer vínculos, lazos, entre bienes y personas. Estos lazos son emociones, recuerdos, vivencias, experiencias, que hacen que un objeto deje de ser únicamente una entidad material, para convertirse en parte de nuestra historia personal, social, vivencial, incorporándolo 
$2^{\text {nd }}$ International Conference Art. Illustration and Visual Culture in Infant and Primary Education
Figura 5. Gráfico ilustrativo del planteamiento del modelo educativo. a nuestras "señas de identidad" propias. Entender el arte contemporáneo desde su dimensión patrimonial significa valorarlo desde su cualidad simbólica y sus posibilidades para generar nuevos significados y establecer vínculos con las personas, construyendo nuevas identidades. Esto supone poner el acento en el individuo, la persona como protagonista en el proceso de patrimonializar un determinado bien, desde la acción y la participación.

Partiendo de estas premisas el modelo que proponemos se orienta a guiar el proceso de apropiación simbólica, el proceso patrimonializador, o bien el proceso de vincularse con un objeto a través de la generación de nuevos significados, nuevos lazos o conexiones.

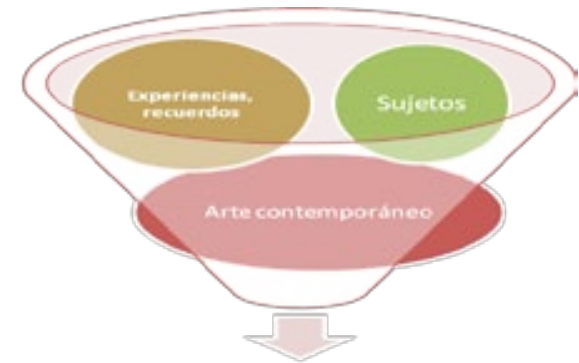

\section{PATRIMONIALIZACIÓN}

Por ello, el modelo que definimos se centra en guiar y propiciar la patrimonialización basándonos en la filosofía descrita, así como en principios de procedimiento, algunos de los cuales resumimos a continuación:

- El aprendizaje significativo desde lo personal a lo global.

- Los participantes son protagonistas a través de un proceso de descubrimiento personal.

- Generar su propia ruta/visión patrimonial del museo, descubriendo y reflexionando sobre los lazos que nos unen a objetos y que nos dotan de identidad.

- La propiedad simbólica y significados de los objetos.

- Uso/experimentación a través de la fotografía, colaboración inter-grupos, ampliando la patrimonialización a entornos más amplios a través de la red social (internet).

Como consecuencia de los hallazgos de nuestro estudio, el modelo educativo que definimos se caracteriza por ser intermediario, guiando el proceso patrimonializador de apropiación simbólica, el proceso de vincularse con un objeto a través de la generación de nuevos significados, nuevos lazos o conexiones. De esta forma, iniciamos una segunda fase de investigación para intervenir aplicando nuestro modelo en museos de arte contemporáneo con todo tipo de destinatarios y capacidades diferentes, desde educación infantil, educación primaria, hasta nuestros mayores, con el objetivo de estudiar la efectividad del mismo para trabajar la normalización a través de la educación patrimonial, luchando por cumplir con las necesidades detectadas derivadas de la primera fase de nuestra investigación.

Fontal Merillas, O. (2003). La Educación Patrimonial. Teoría y práctica en el aula, el museo e internet. Gijón: Trea.

Fontal Merillas, O. (2004). Museos de arte y TICs: usos, tipologías, ejemplos y derivaciones. En Vera Muñoz, M.I. y Pérez, D. (Coord.), Formación de la ciudadanía: las TICs y los nuevos problemas. Alicante: Asociación Universitaria de Profesores de Didáctica de las Ciencias Sociales.

Gustave, C. (1855). Manifiesto del realismo. París.

Mateos, S. M. (2008). La comunicación global del patrimonio cultural. Gijón: Trea. 